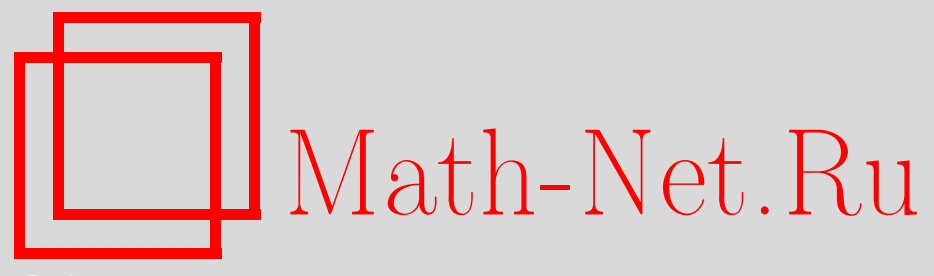

А. Н. Копежанова, Е. Д. Нурсултанов, Л.-Е. Перссон, О неравенствах для преобразования Фурье функций из пространств Лоренца, Матем. заметки, 2011, том 90, выпуск 5, 785-788

DOI: https://doi.org/10.4213/mzm9270

Использование Общероссийского математического портала Math-Net.Ru подразумевает, что вы прочитали и согласны с пользовательским соглашением http://www . mathnet.ru/rus/agreement

Параметры загрузки:

IP: 54.196 .121 .252

26 апреля 2023 г., 04:08:45

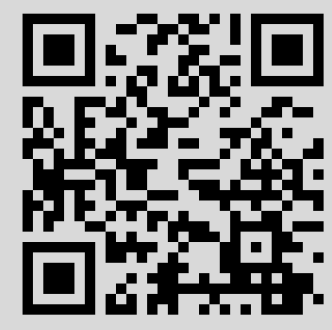




\section{О неравенствах для преобразования Фурье функций из пространств Лоренца}

\section{А. Н. Копежанова, Е. Д. Нурсултанов, Л.-Е. Перссон}

1. Пусть

$$
\widehat{f}(t)=\frac{1}{\sqrt{2 \pi}} \int_{-\infty}^{\infty} f(x) e^{-i t x} d x, \quad x \in \mathbb{R},
$$

- преобразование Фурье функции $f \in L_{1}(\mathbb{R})$. Хорошо известны неравенства, связывающие интегральные свойства функций и ее преобразования Фурье.

Пусть $1<p<2, p^{\prime}=p /(p-1)$ и $0<q \leqslant \infty$. Тогда имеют место неравенства

$$
\begin{aligned}
\|\widehat{f}\|_{L_{p^{\prime}}(\mathbb{R})} & \leqslant c_{1}\|f\|_{L_{p}(\mathbb{R})}, \\
\|\widehat{f}\|_{L_{p^{\prime}, q}(\mathbb{R})} & \leqslant c_{2}\|f\|_{L_{p, q}(\mathbb{R})},
\end{aligned}
$$

где $L_{p, q}(\mathbb{R})$ - классическое пространство Лоренца. Эти неравенства называют неравенствами Хаусдорфа-Юнга и Харди-Литтлвуда-Стейна, соответственно [1]. Имеются аналогичные неравенства для преобразования Фурье на торе $[0,1]$, т.е. для $\widehat{f}=\left\{\widehat{f}_{n}\right\}$, где $\widehat{f}_{n}-$ коэффициенты Фурье по некоторой ортонормированной системе.

Пусть $\omega-$ неотрицательная функция на $[0, \infty]$. Обобщенное пространство Лоренца $\Lambda_{q}(\omega, \mathbb{R})$ - это множество всех измеримых функций $f$ на $\mathbb{R}$ таких, что:

- если $0<q<\infty$, то

$$
\|f\|_{\Lambda_{q}(\omega, \mathbb{R})}=\left(\int_{0}^{\infty}\left(f^{*}(t) \omega(t)\right)^{q} \frac{d t}{t}\right)^{1 / q}<\infty
$$

- если $q=\infty$, то

$$
\|f\|_{\Lambda_{\infty}(\omega, \mathbb{R})}=\sup _{t>0} f^{*}(t) \omega(t)
$$

где $f^{*}$ - невозрастающая перестановка функции $f$.

В данной работе изучаются верхние и нижние оценки нормы преобразования Фурье в обобщенных пространствах Лоренца. Получены в некотором смысле обратные к (1) и (2) неравенства для преобразования Фурье на $\mathbb{R}$ и на торе $[0,1]$.

Пусть $\delta>0$ и $\omega(t)$ - неотрицательная функция на $[0, \infty)$. Классы функции $A_{\delta}$ и $B_{\delta}$ определим следующим образом:

$$
\begin{aligned}
& A_{\delta}=\left\{\omega(t): \omega(t) t^{-1 / 2-\delta}-\text { возрастающая функция, } \omega(t) t^{-(1-\delta)}-\text { убывающая функция }\right\}, \\
& B_{\delta}=\left\{\omega(t): \omega(t) t^{-\delta}-\text { возрастающая функция, } \omega(t) t^{-1+\delta}-\text { убывающая функция }\right\} .
\end{aligned}
$$

Тогда классы $A$ и $B$ определяются следующим образом:

$$
A=\bigcup_{\delta>0} A_{\delta}, \quad B=\bigcup_{\delta>0} B_{\delta} .
$$

Отметим, что класс $B$ содержит в себе класс $A$.

Верна следующая теорема.

Теорема 1. Пусть $0<q \leqslant \infty u \omega(t)$ принадлежит классу А. Тогда имеют место следующие неравенства:

$$
c_{1}\|\bar{f}\|_{\Lambda_{q}(\omega, \mathbb{R})} \leqslant\|\widehat{f}\|_{\Lambda_{q}(\mu, \mathbb{R})} \leqslant c_{2}\|f\|_{\Lambda_{q}(\omega, \mathbb{R})},
$$

где $\bar{f}(t)=(1 / t)\left|\int_{0}^{t} f(s) d s\right|, \mu(t)=t \omega(1 / t)$.

(C) А. Н. КоПЕЖАнова, Е. Д. Нурсултанов, Л.-Е. ПерССон, 
Неравенства в (3) и далее понимаются в том смысле, что если правая часть неравенства конечна, то и левая часть так же конечна, и выполняется соответствующее неравенство.

В частности, когда $w(t)=t^{1 / p}, 1<p<2$, то

$$
c_{1}\|\bar{f}\|_{L_{p, q}} \leqslant\|\widehat{f}\|_{L_{p^{\prime} q}} \leqslant c_{2}\|f\|_{L_{p, q}} .
$$

Отметим, что левое неравенство в (4) также следует из результатов работ [2], [3], где используются сетевые пространства.

Будем говорить, что функция $f$, определенная на $\mathbb{R}$, обобщенно-монотонная, если найдется некоторая константа $M>0$, что верно следующее неравенство:

$$
|f(x)| \leqslant M \frac{1}{2 x}\left|\int_{-x}^{x} f(t) d t\right|, \quad x>0 .
$$

СлеДСТвиЕ 1. Пусть $f$ - обобщенно-монотонная функиия, $0<q \leqslant \infty u \omega(t)$ принадлежит классу $A$. Тогда для того, чтобъ $\widehat{f} \in \Lambda_{q}(\mu, \mathbb{R})$ необходимо и достаточно, чтобъ $f \in \Lambda_{q}(\omega, \mathbb{R})$, и выполнялосъ соотношение

$$
\|f\|_{\Lambda_{q}(\omega, \mathbb{R})} \approx\|\widehat{f}\|_{\Lambda_{q}(\mu, \mathbb{R})} .
$$

2. Пусть $f$ периодическая функция с периодом 1 и интегрируемая на $[0,1]$ и $\Phi=$ $\left\{\varphi_{k}\right\}_{k=1}^{\infty}-$ ортонормированная система,

$$
a_{k}=a_{k}(f)=\int_{0}^{1} f(x) \overline{\varphi_{k}(x)} d \mu, \quad k \in \mathbb{N},
$$

коэффициентами Фурье функции $f$ по системе $\Phi=\left\{\varphi_{n}\right\}_{n=1}^{\infty}$.

Ортонормированную систему $\Phi=\left\{\phi_{k}(x)\right\}_{k=1}^{\infty}$ назовем регулярной, если существует константа $B$ такая, что:

1) для любого отрезка $е$ из $[0,1]$ и $k \in \mathbf{N}$ верно

$$
\left|\int_{e} \phi_{k}(x) d x\right| \leqslant B \min \left(|e|, \frac{1}{k}\right)
$$

2) для любого отрезка $w$ из $\mathbf{N}$ и $t \in[0,1]$

$$
\left(\sum_{k \in w} \phi_{k}(\cdot)\right)^{*}(t) \leqslant B \min \left(|w|, \frac{1}{t}\right)
$$

где $\left(\sum_{k \in w} \phi_{k}(\cdot)\right)^{*}(t)$ - невозрастающая перестановка функции $\sum_{k \in w} \phi_{k}(x)$.

Примерами регулярной системы являются: все тригонометрические системы, система Уолша и система Прайса и др.

Tеорема 2. Пусть $\Phi=\left\{\varphi_{n}\right\}_{n=1}^{\infty}-$ регулярная система и $f \stackrel{\text { П.в. }}{=} \sum_{n=1}^{\infty} a_{k} \phi_{n}$. Пусть $0 \leqslant q \leqslant \infty u \omega(t)$ принадлежит классу $B$. Тогда

$$
\left(\int_{0}^{1}(\bar{f}(t) \omega(t))^{q} \frac{d t}{t}\right)^{1 / q} \leqslant c_{1}\left(\sum_{k=1}^{\infty}\left(a_{k}^{*} \mu(k)\right)^{q} \frac{1}{k}\right)^{1 / q},
$$

где $\bar{f}(t)=(1 / t)\left|\int_{0}^{t} f(s) d s\right|, \mu(k)=k \omega(1 / k), a_{k}^{*}$ - невозрастающая перестановка коэффициентов $\left\{a_{k}\right\}$. 
Отметим, что если в теоремах 1,2 вместо $\bar{f}(t)$ поставить $(1 / t) \int_{0}^{t}|f(s)| d s$ или $|f(t)|$, или $f^{*}(t)$, то соответствующие неравенства не сохраняются (построены примеры).

Будем говорить, что последовательность комплексных чисел $\left\{a_{k}\right\}_{k \in \mathbb{N}}$ обобщенно-монотонная, если найдется такая константа $M>0$, что

$$
\left|a_{n}\right| \leqslant M \frac{1}{n}\left|\sum_{k=1}^{n} a_{k}\right|, \quad n \in \mathbb{N} .
$$

В работе [4] для $\omega \in A$ получено неравенство

$$
\left(\sum_{k=1}^{\infty}\left(a_{k}^{*} \mu(k)\right)^{q} \frac{1}{k}\right)^{1 / q} \leqslant\|f\|_{\Lambda_{q}(\omega,[0,1])}:=\left(\int_{0}^{1}\left(f^{*}(t) \omega(t)\right)^{q} \frac{d t}{t}\right)^{1 / q} .
$$

Используя это неравенство и теорему 2, доказывается следующее утверждение.

Следствие 2 . Пусть $1 \leqslant q \leqslant \infty, \Phi=\left\{\varphi_{k}\right\}_{k=1}^{\infty}-$ регулярная система, $f \stackrel{\text { п.в. }}{=} \sum_{k=1}^{\infty} a_{k} \varphi_{k}$, $\omega(t) \in B \backslash A$. Если $f$ - обобщенно-монотонная функция либо $\left\{a_{k}\right\}$ - обобщенно-монотонна, то верно соотношение

$$
\|f\|_{\Lambda_{q}(\omega,[0,1])} \approx\left(\sum_{k=1}^{\infty}\left(a_{k}^{*} \mu(k)\right)^{q} \frac{1}{k}\right)^{1 / q}
$$

где $\mu(k)=k \omega(1 / k)$

Условие принадлежности веса $\omega$ классу $B \backslash A$ в случае $\omega(t)=t^{1 / p}$ индуцирует условие $2<p<\infty$. Таким образом, из следствия 2 следует, что при $1<p<2$ и при условии обобщенно-монотонности $\left\{a_{k}\right\}$ для $f=\sum a_{k} \varphi_{k}$ имеет место соотношение

$$
\|f\|_{L_{p}}^{p} \approx \sum_{k=1}^{\infty} k^{p-2}\left|a_{k}\right| .
$$

В случае $p<2$ построен пример тригонометрического ряда с обобщенно-монотонными коэффициенами, для которого соотношение (5) не выполняется. Это говорит о существенности условия $\omega \in B \backslash A$ (идея построения примера принадлежит М. И. Дьяченко).

Теорема 3. Пусть $\Phi=\left\{\varphi_{k}\right\}_{k=1}^{\infty}-$ регулярная система, $1 \leqslant q \leqslant \infty u f \stackrel{\text { п.в. }}{=} \sum_{k=1}^{\infty} a_{k} \varphi_{k}$. Если $\omega(t)$ принадлежит классу $B$, то

$$
c_{1}\left(\sum_{k=1}^{\infty}\left(\bar{a}_{k} \mu(k)\right)^{q} \frac{1}{k}\right)^{1 / q} \leqslant c_{2}\|f\|_{\Lambda_{q}(\omega,[0,1])} \leqslant c_{2}\left(\sum_{k=1}^{\infty}\left(\left|k \Delta a_{k}\right| \mu(k)\right)^{q} \frac{1}{k}\right)^{1 / q},
$$

где $\bar{a}_{k}=(1 / k)\left|\sum_{m=1}^{k} a_{m}(f)\right| u \Delta a_{k}=a_{k}-a_{k+1} u \mu(k)=k \omega(1 / k)$.

СледСтвие 3. Пусть $\Phi=\left\{\varphi_{k}\right\}_{k=1}^{\infty}-$ регулярная система, $f \stackrel{n . \bullet .}{=} \sum_{k=1}^{\infty} a_{k} \varphi_{k} u \omega(t) n p u$ надлежит классу $B$. Если последовательность комплексных чисел $\left\{a_{k}\right\}$ удовлетворяет условию: найдутся такие $C>0, d \geqslant 1$, что

$$
\sum_{k=n}^{\infty}\left|\Delta a_{k}\right| \leqslant C \bar{a}_{d n}, \quad n \in \mathbb{N}
$$

то верно соотношение

$$
\|f\|_{\Lambda_{1}(\omega,[0,1])} \approx \sum_{k=1}^{\infty}\left|a_{k}\right| \omega\left(\frac{1}{k}\right) .
$$

Заметим, что класс последовательностей, удовлетворяющих условию (6), шире класса $G M\left({ }_{*} \beta\right)$, введенного в работе [5]. 


\section{СПИСОК ЦИТИРОВАННОЙ ЛИТЕРАТУРЫ}

[1] E. M. Stein, Trans. Amer. Math. Soc., 83 (1956), 482-492. [2] Е. Д. Нурсултанов, Докл. PAH, 361:5 (1998), 597-599. [3] E. Nursultanov, S. Tikhonov, "Net spaces and boundedness of integral operators", J. Geom. Anal., 2010, Online First. [4] L.-E. Persson, Acta Math. Acad. Sci. Hungar., 27:3-4 (1976), 267-280. [5] M. Dyachenko, S. Tikhonov, Topics in Classical Analysis and Applications in Honor of Daniel Waterman, World Sci. Publ., Hackensack, NJ, 2008, 88-101.

\section{Е. Д. Нурсултанов}

Поступило

E-mail: er-nurs@yandex.ru 